\title{
Técnicas de relación con la prensa en la gestión de la comunicación organizacional: el caso del Consejo Regulador del Brandy de Jerez
}

Marta Pulido ${ }^{1}$ I martapulido@us.es

UNIVERSIDAD De SEVILLA

Resumen: Las relaciones públicas describen un proceso directivo que se encarga de gestionar la comunicación que las organizaciones establecen con los diferentes públicos de su entorno. El objetivo principal de esta investigación es demostrar el grado de importancia que el empleo de técnicas de relaciones con los medios de comunicación social tiene en la gestión de la comunicación organizacional. Para responder a este objetivo, se analiza el comportamiento comunicacional del Consejo Regulador del Brandy de Jerez durante sus veinte años de historia, utilizando una triangulación metodológica fundamentada en el método del estudio de caso y el análisis de contenido.

Palabras clave: Comunicación organizacional, medios de comunicación, técnicas de relaciones públicas.

Abstract: Public relations describe a management process that takes charge managing the relations that the organizations establish with its different publics. The principal aim of this investigation is to demonstrate that the employment of technologies of relations with the mass media is one of the axes of the institutional communication. According to this aim, we select as object of study the Consejo Regulador del Brandy de Jerez, and there we use a mixed methodology that combines in addition the employment of the method of the study of case and the analysis of content.

Key words: Corporate communication, mass media, public relations techniques

\footnotetext{
${ }^{1}$ Doctora en Comunicación. Licenciada en Publicidad y Relaciones Públicas. Profesora del Departamento de Comunicación Audiovisual, Publicidad y Literatura de la Universidad de Sevilla. Directora de Comunicación de la Organización Internacional de Ceremonial y Protocolo (OICP). Presidenta de la Asociación Universitaria de Relaciones Públicas y Protocolo de Andalucía (ARPPA).
} 


\title{
1. Introducción y objetivos
}

Todas las entidades, físicas o jurídicas, comunican. Es imposible no comunicar (Fernández López, 2007:28). El surgimiento de la sociedad del conocimiento hace que las organizaciones tengan que hacer frente a nuevas necesidades comunicativas en su entorno (Almansa Martínez, 2005:9). Es entonces cuando la comunicación debe ser concebida como un proceso planificado y consciente para convertirse en una herramienta eficaz de gestión organizacional (empresarial) que favorezca la consecución de las metas y los objetivos corporativos (Cees Van Riel, 2001: 1-2) a través de la gestión estratégica de las relaciones entre la organización y sus públicos. Este proceso de gestión exige la utilización, entre otras, de técnicas de relaciones con los medios de comunicación social.

Las relaciones con los medios de comunicación social son uno de los aspectos más relevantes que habitualmente se destacan cuando se habla de la gestión de la comunicación organizacional y la planificación estratégica de las relaciones públicas. El éxito de estas técnicas radica en la capacidad que, como técnicas específicas de relaciones públicas, tienen de gestionar las relaciones entre una organización y los medios de comunicación a fin de situar en la agenda mediática y la opinión pública los mensajes corporativos de la organización.

Asimismo, como afirma Xifra (2007:69-70), las relaciones con los medios representan la parte más visible del trabajo el profesional de las relaciones públicas llegando a acaparar, en algunas organizaciones, más recursos económicos y temporales que cualq uier otra área comunicativa.

El nacimiento de las relaciones públicas como tal viene ligado a las relaciones con los medios de comunicación social y a la gestión de la publicity. En palabras de Xifra (2007: 70):

\begin{abstract}
La evolución de la práctica de las relaciones públicas demuestra la importancia de las relaciones con la prensa. El modelo del agente de prensa es precisamente un modelo de relaciones con los medios de comunicación plenamente vigente, aunque las artimañas de finales del siglo XIX para conseguir espacio informativo ya no sean las mismas. En efecto, en la actualidad, ya no se pactan entrevistas sin validar la certeza de las informaciones transmitidas, la solidez de las pruebas y de los datos aportados. Transparencia y competencia en la difusión de las informaciones a los mass media son, pues, dos de los pilares de una comunicación eficaz y respetuosa con las necesidades de los periodistas e, indirectamente, de la población general.
\end{abstract}

Además, la propia evolución de la profesión periodística ha favorecido la relación entre las organizaciones y los medios de comunicación ya que el auge actual del denominado periodismo de fuentes, evidencia que las agencias de relaciones públicas se están convirtiendo en la principal fuente de los medios informativos. Como afirma Xifra (2007: 7071):

La profesión de periodista también ha cambiado con el tiempo. La tendencia a la especialización temática por parte de los profesionales, especialmente de la prensa escrita, y la creciente concentración registrada en la propiedad de los medios de comunicación son algunos de los 
factores que contribuyen a redibujar el panorama del periodismo actual. (...) Así pues, los cambios en las rutinas periodísticas han acabado por favorecer la acción de los profesionales de las relaciones públicas, que se han convertido en la principal fuente de información de los medios de comunicación social (...).

Grunig (2001:337) coincide en destacar la posición central que la relación con los medios ocupa tanto en el desarrollo histórico como en el ejercicio actual de las relaciones públicas, pero advierte sobre la importancia justa que la relación con la prensa debe tener actualmente en los programas de relaciones públicas y establece:

(...) ciertamente, las relaciones públicas evolucionaron a partir de los esfuerzos para influir en la cobertura de la prensa de organizaciones e individuos. Sin embargo, en la actualidad, las relaciones con los medios de comunicación social deberían ser consideradas uno más entre los programas importantes de relaciones públicas enfocados a públicos especializados.

Como se puede observar, Grunig representa una corriente de autores que tiende a moderar el entusiasmo por el uso de técnicas de relación con los medios de comunicación social a la hora de gestionar procesos de relaciones públicas. Corriente a la que se suma, en el ámbito español, Castillo que afirma que, a pesar de que las relaciones con los medios han venido siendo estudiadas como la actividad fundamental de las relaciones públicas externas, deben ser concebidas como "una de las facetas más importantes en la comunicación pero ni la exclusiva ni se agota en ella" (2010:105).

En este contexto, podemos afirmar que, a pesar de la literatura consultada, existen escasos trabajos orientados a demostrar, a través de un proceso de contrastación empírica, el grado de importancia que el empleo de técnicas específicas de relación con la prensa tiene frente a otras técnicas comunicativas a la hora de gestionar la comunicación corporativa. En este sentido, este trabajo tiene como objetivo principal analizar el comportamiento comunicacional del Consejo Regulador del Brandy de Jerez (en adelante CRBJ) durante sus 20 años de historia (entre 1989 y 2008) tratando de averiguar en qué medida se utilizan las técnicas de relación con los medios de comunicación a la hora de gestionar la comunicación organizacional frente a otras técnicas de relaciones públicas.

Para poder alcanzar este objetivo principal se establecen los siguientes objetivos secundarios:

- Establecer un marco teórico que explique la gestión de la comunicación organizacional como un proceso de planificación estratégica orientado a establecer un sistema de relaciones mutuamente beneficioso entre una organización y los públicos de su entorno.

- Observar qué papel juegan en dicho proceso las técnicas específicas de relaciones públicas y, en concreto, las técnicas de relación con los medios de comunicación social.

- Identificar las técnicas de relación con la prensa más referenciadas por los autores clásicos de las relaciones públicas y realizar una breve aproximación conceptual a cada una de ellas. 
- Diseñar y aplicar un diseño metodológico que nos permita determinar y analizar el comportamiento comunicacional del CRBJ para determinar en qué grado:

- se utilizan las técnicas de relación con los medios de comunicación frente a otras técnicas de relaciones públicas y,

- se contemplan los periodistas como públicos específicos a la hora de gestionar la comunicación de la organización objeto de estudio.

\section{Marco teórico}

2.1. La gestión de la comunicación organizacional como proceso de planificación estratégica de las relaciones públicas

Las relaciones públicas describen un proceso directivo que se encarga de gestionar la comunicación organizacional a través de la identificación, establecimiento y mantenimiento de relaciones, mutuamente beneficiosas, entre la organización y los diferentes públicos de su entorno. Para Grunig (2000: 35-36), este proceso de gestión debe estar concebido desde un planteamiento estratégico en relación a la búsqueda de la excelencia en las organizaciones.

Ya Bernays (Xifra 2003:36) ya describía la actividad de las relaciones públicas como proceso estratégico cuando indicaba las ocho etapas fundamentales de un programa de relaciones públicas: definir los objetivos, investigar a los públicos, modificar los objetivos para conseguir los fines que la investigación señala como alcanzables, decidir la estrategia particular del caso, crear y desarrollar el mensaje, programar detalladamente la acción y la organización necesaria para ejecutarla, especificar el plan táctico y su programación temporal y desarrollar las tácticas.

Desde entonces, diferentes autores han venido contribuyendo al desarrollo de diversos modelos teórico-prácticos encaminados a tratar de explicar dicho proceso, llegando a recoger Noguero (1995: 73-127) hasta veintiún modelos que explican el proceso de las relaciones públicas y que finalmente sintetiza mediante la variable de su función en torno a tres tipos de funciones: funciones teórico/doctrinales, funciones operativo/ aplicativas y funciones mixtas o híbridas.

Para Cutlip y Center (2001:444) las relaciones públicas consisten en un proceso de planificación y programación en el que hay que tomar decisiones estratégicas sobre lo que hay que hacer y el orden en el que se debe hacer en respuesta o anticipándose a un problema u oportunidad. Es decir, una vez se ha definido el problema u oportunidad de relaciones públicas a través de la investigación y el análisis, ha de idearse una estrategia que sea capaz de hacer frente a dicho problema "o capitalizar la oportunidad". 
Desde esta perspectiva, establecen que la gestión de la comunicación organizacional debe sustentarse en el pensamiento estratégico que implica "predecir o establecer un futuro estado de metas deseado y determinar las fuerzas que ayudarán o impedirán avanzar hacia la meta, y formular un plan para lograr el estado deseado" (Cutlip y Center, 2001:445).

\subsection{Los elementos de la planificación estratégica y las técnicas de relaciones públicas}

Partiendo de los postulados de la dirección por objetivos, un plan de relaciones públicas debe identificar lo que hay que hacer, por qué y cómo, componiéndose de los siguientes ocho elementos esenciales (Wilcox, Cameron y Xifra, 2009:196-197):

- Situación: cuando se inicia un programa de relaciones públicas la organización está respondiendo a una de las tres situaciones fundamentales que pueden crear esta necesidad: resolver una situación que le afecta negativamente, acometer un determinado proyecto o, fortalecer su reputación y/o el respaldo de su entorno.

- Objetivos: enmarcados en los objetivos generales de la organización, los objetivos del plan de relaciones públicas deben ser realistas, alcanzables y cuantificables. Pueden ser informativos (cuando pretenden informar para concienciar de una determinada situación) y de motivación (orientados a cambiar actitudes).

- Público: una de las claves de un programa de relaciones públicas eficaz es identificar adecuadamente a cada uno de los públicos de la organización atendiendo a la situación de partida y los objetivos planteados.

- Estrategia: establecer la estrategia implica determinar el plan global de juego a cerca de cómo se van a alcanzar los objetivos inicialmente propuestos.

- Tácticas: son las iniciativas puntuales que permiten adaptar la estrategia al terreno de juego sorteando posibles obstáculos que pueden surgir en la implantación del programa de relaciones públicas.

- Calendario: implica decidir cuándo debe realizarse cada una de las secuencias de la campaña.

- Presupuesto: cada uno de los elementos de la planificación realizada debe ajustarse a los recursos económicos del departamento de relaciones públicas.

- Evaluación: implica comprar los objetivos inicialmente planteados con los resultados conseguidos. Unos objetivos establecidos correctamente facilitan el diseño de la fase de investigación. 
Las técnicas de relaciones públicas cumplen un papel fundamental en este proceso de planificación estratégica ya que son los mecanismos a través de los cuales los relacionistas públicos gestionan la transmisión de determinados mensajes organizacionales a los públicos identificados para implementar la estrategia previamente definida en la que a su vez se enmarcan.

Las técnicas son, pues, herramientas a través de las cuales los profesionales de las relaciones públicas pueden vehicular los mensajes adaptándolos a las necesidades de cada uno de los públicos definidos. No deben ser confundidas con las tácticas, pues si bien una técnica no siempre se utilizará tácticamente, una táctica es por definición una técnica aplicada por la inmediatez de reacción que exige un ajuste del programa la realidad.

Así, obedeciendo a las líneas estratégicas marcadas por el plan de relaciones públicas, el profesional de las relaciones públicas hará uso de unas u otras técnicas en pos de gestionar de forma eficaz la comunicación de la organización para la que trabaje (Noguero, 1988: 144).

En este sentido, las técnicas de relaciones públicas se reúnen fundamentalmente en torno a cuatro grandes bloques (Xifra, 2007 y 2011):

- Técnicas de relaciones públicas internas: aluden a aquellas técnicas encaminadas a gestionar las relaciones entre la organización y sus públicos internos (trabajadores, mandos intermedios, directivos y comité de empresa). En determinados programas específicos de relaciones públicas internas pueden incluso contemplarse como público interno los familiares de los trabajadores y los accionistas, aún no perteneciendo al organigrama de la corporación.

- Técnicas con los medios de comunicación social: agrupan aquellas técnicas específicas orientadas a gestionar las relaciones entre la organización y los medios de comunicación con el objetivo de que el punto de la voz de la organización pueda ser tenida en cuenta por la prensa y ser trasladada a la opinión pública. Podríamos considerar que los medios de comunicación son un público doble: por un lado son concebidos como públicos en sí mismos en programas estratégicos de relaciones públicas pero, al mismo tiempo, son intermediarios necesarios para llegar a la opinión pública general.

- Técnicas de relación con la comunidad: son aquellas técnicas que tienen como finalidad situar directamente en determinados grupos sociales del entorno de la organización los mensajes corporativos previamente definidos en cada programa de relaciones públicas.

- Técnicas transversales: definen aquellas técnicas que, siendo utilizadas con asiduidad en programas estratégicos de gestión de la comunicación organizacional, no son exclusivas de las relaciones públicas tales como las alocuciones en foros de interés. 


\title{
2.3. Las técnicas de relación con los medios de comunicación social
}

El uso de técnicas de relación con la prensa en programas estratégicos de relaciones públicas tiene como objetivo principal crear un clima de confianza y respeto entre la organización y los medios de comunicación, aun asumiendo la divergencia de intereses existente entre el relacionista público y el periodista como público específico de la organización. En palabras de Cutlip y Center:

\begin{abstract}
Los profesionales encargados de tratar con los medios y con las personas clave para el acceso a ellos, deben establecer y mantener relaciones de respeto mutuo y confianza. La relación, aun siendo una relación de beneficio mutuo es en esencia una relación de adversarios, ya que los periodistas y los relaciones públicas no están en el mismo negocio, y a menudo no tienen las mismas metas de comunicación.
\end{abstract}

Para crear este clima de confianza, respeto y beneficio mutuo, la organización debe tener presente una serie de condicionantes que deben regir su relación con la prensa (Castillo, 2010:107-108):

- La organización debe tener ánimo de informar pero sin olvidar sus propios intereses corporativos

- Debe establecerse una comunicación única y abierta con los periodistas, siempre positiva, proporcionando una colaboración ágil y eficaz, cada vez que los medios lo soliciten

- La información debe ser siempre veráz y

- La transparencia informativa debe erigirse como el modelo de comportamiento a seguir

Desde esta perspectiva, la mayor parte de los autores consultados (Grunig, 2000; Cutlip y Center 2000; Wilcox, Autt, Agee y Cameron, 2000; Wilcox, Cameron y Xifra, 2009; Castillo, 2010; Xifra, 2007 y 2011) coinciden en destacar la nota de prensa, la rueda de prensa, el dossier de prensa y otros encuentros con la prensa como las técnicas específicas más relevantes de relación con los medios de comunicación.

\subsubsection{La nota de prensa}

La nota de prensa es un escrito breve que utiliza el lenguaje periodístico e imita el formato tradicional de la noticia para transmitir información a los medios de comunicación. Se redacta con el objetivo de que sea publicada por el medio con la menor manipulación posible y su finalidad es trasladar a los medios de comunicación el punto de vista de la organización.

\subsubsection{La rueda o conferencia de prensa}

Es una reunión convocada por la organización para proporcionar a los medios invitados información de especial relevancia a través de sus portavoces de primera mano. Combina la información verbal con la no verbal y su objetivo es generar un clima de diálogo que fomente 
las relaciones interpersonales con los informadores y consiga situar a la organización como una fuente de información fidedigna.

\subsubsection{El dossier de prensa}

Es un documento versátil que resume la información general de la organización y que se envía a los medios bien bajo demanda o bien con motivo de una rueda de prensa, presentación u otro encuentro con la prensa. Su objetivo es facilitar la elaboración de una información más amplia por parte de los periodistas.

\subsubsection{Otros encuentros con la prensa}

Además de la rueda de prensa tradicional, suelen emplearse otros encuentros con la prensa con la finalidad de gestionar la bidireccionalidad de la comunicación entre la organización y los medios de comunicación tales como entrevistas, desayunos o almuerzos de trabajo, reuniones informales, charlas coloquio y presentaciones o prueba de productos.

\section{Metodología}

La metodología debe ser entendida como el estudio (descripción, explicación y justificación) de los métodos empleados en una investigación (González Río, 1997:16) y una de las primeras consideraciones del investigador a la hora de abordarla es su carácter cualitativo o cuantitativo.

Partiendo del contexto teórico que plantea los beneficios del empleo integrado y eficaz de los métodos cualitativos y cuantitativos, que implica el concepto de triangulación entre-métodos (González Río, 1997:283), el diseño de la presente investigación se basa en la aplicación complementaria de dos métodos: uno cualitativo (Pérez Serrano, 2004) como método principal, el estudio de casos: diseño de caso único, y uno cuantitativo como método complementario, el análisis de contenido (Corbetta, 2007).

La recolección de datos se hace a través del diseño de diferentes herramientas basadas en las técnicas de la observación, la entrevista cualitativa y las fuentes de datos secundarios.

Los datos secundarios utilizados son los documentos públicos y/o legales y los documentos bibliográficos. A su vez, entre los documentos públicos utilizados destacan por su rentabilidad informativa las memorias de actividad anuales correspondientes al período investigado. Desde el punto de vista de la investigadora, su importancia es tal que, para el análisis, rendimiento y aprovechamiento de la información contenida, es sometido al método del análisis de contenido como método complementario.

Para llevar a cabo el análisis de contenido se diseñan las siguientes herramientas metodológicas: 
- Clasificación y organización por lustros,

- Lectura sistemática aplicando una unidad de análisis: texto descriptivo de cada unas de las actividades de comunicación contempladas en el área de comunicación de cada una de las memorias analizadas,

- Clasificación y organización por lustros de cada una de las actividades de comunicación en una matriz de datos general,

- Aplicación a la Matriz general de datos de los factores de frecuencia AF1 (aplicado a aquellas acciones que solo se emplean una vez por la organización estudiada) y AF+ (aplicado a aquellas acciones que se emplean más de una vez). La aplicación de estos factores permite identificar el peso específico de cada acción de comunicación dentro de cada lustro analizado.

- Triangulación de los datos obtenidos de las memorias con los datos obtenidos a través de una entrevista en profundidad, implementando la matriz de datos general.

- Tomando como punto de partida las técnicas de relaciones públicas identificadas en la obra de Xifra (2007), se diseña un índice A relativo a la identificación de técnicas específicas de relaciones públicas, aspecto clave sobre el que se centra el presente artículo. Este Índice se aplica sobre la matriz de datos general implementada, logrando así identificar cada acción de comunicación descrita en la memoria de actividades,

- Tabulación de los datos obtenidos en las tablas expuestas en el apartado de resultados del análisis.

\section{Resultados del análisis}

En primer lugar, tal y como puede observarse en la tabla 1, el análisis revela el registro de un total de 367 acciones que definen el comportamiento comunicacional llevado a cabo por el CRBJ entre 1989 y 2008. De esas 367 acciones registradas, 311 se identifican con el empleo de técnicas de relaciones públicas, 36 se registran como otras técnicas de comunicación (como la publicidad institucional) y 20 se registran sin datos suficientes para identificar la técnica empleada.

Estos datos evidencian que el comportamiento comunicacional descrito por la organización objeto de estudio se sustenta en un $84,74 \%$ en el empleo de técnicas de relaciones públicas frente al 9,81\% que representan el resto de técnicas de comunicación empleadas.

En segundo lugar, es destacable cómo los resultados del análisis nos permiten observar un claro predominio de las acciones de frecuencia mayor a uno $(\mathrm{AF}+)$. Lo que permitiría potenciar los perfiles que estas acciones muestran. Resulta relevante destacar, que el mayor número de acciones AF1 se registran en el lustro 1, de donde se podría extraer un cierto 
desconcierto en el comportamiento comunicacional del CRDEBJ en sus orígenes, reacción por otro lado natural si atendemos a que el primer lustro supone la puesta en marcha de la organización como tal y que, tal vez, los primeros años las acciones no demuestran un camino claramente identificado. Este hecho explicaría un gran número de acciones que se comienzan y cuyo resultado no permite prever de cara a la organización si su repetición sería corporativamente rentable.

Este hecho se ratifica si se observan los resultados del análisis correspondiente a los lustros 2, 3 y 4, cuyos datos revelan que las acciones de frecuencia 1 (AF1) descienden frente a los de las acciones de mayor frecuencia $(\mathrm{AF}+)$. Este incremento puede interpretarse como una adquisición progresiva de madurez en el aspecto comunicacional por cuanto podrían significar que se repiten con mayor frecuencia aquellas acciones cuyo funcionamiento ha ido siendo comprobado durante los primeros años de vida del CRBJ.

En tercer lugar, la tabla 2 arroja los resultados globales por lustros del análisis atendiendo al registro de acciones identificadas con técnicas de relaciones públicas, siendo especialmente interesante la comparativa en el porcentaje de acciones identificadas por técnicas de relaciones públicas frente al resto, tal y como destacábamos en párrafos anteriores.

Atendiendo a los porcentajes que se observan en la tabla 2, el mayor número de acciones se registran como técnicas de relaciones del CRBJ con la comunidad en un 32,97\%, erigiéndose así claramente como la técnica más utilizada por la organización en los lustros 1, 2, 3 y 4.

El siguiente puesto lo ocupan las acciones que se enmarcan en las técnicas transversales de relaciones públicas, que representan el 20,71 \% del total de acciones registradas. Este dato precede al 20,16 \% referente al total de acciones registradas como técnicas de relación con los medios de comunicación social que, desde esta perspectiva, se ubican en el tercer puesto del ranking en relación a las técnicas de relaciones públicas utilizadas por el CRBJ entre 1989 y 2008 , seguidas por el $10,90 \%$ que representa el empleo de técnicas de relación con los públicos internos.

Finalmente, encontramos las acciones registradas como otras técnicas de comunicación que representan el 9,81\% del total de registros.

En cuarto lugar, en la tabla 3 se presentan los resultados obtenidos del análisis pormenorizado atendiendo a cada una de las diferentes categorías de técnicas de relación con los medios de comunicación social

En este sentido, el análisis revela que la técnica de relación con la prensa más utilizada es la rueda de prensa $(28 \%)$, seguida por la nota de prensa $(26,67 \%)$, la visita de prensa $(41,17 \%)$ y la atención bajo demanda de información (8\%). 
Una de las técnicas más específicas observadas, dada la naturaleza de la organización objeto de estudio, son las catas dirigidas que, según la clasificación de técnicas establecida por Xifra (2007) podría englobarse bajo la categoría ensayo de productos u otros encuentros con la prensa, tal y como ya mencionábamos en el marco teórico. La cata dirigida destinada a periodistas supone un $6,67 \%$ del total de técnicas de relaciones públicas empleadas por el CRBJ.

Especial mención merece en esta tabla el registro de técnicas genéricas de relación con la prensa, ya que un $14,67 \%$ de las acciones registradas se manifestaban de forma general, sin especificar, como relaciones con los medios de comunicación. Asimismo, llama la atención la escasa acogida del dosier de prensa como técnica de relación con la prensa. Podría intuirse que la memoria anual que legalmente los consejos reguladores están obligados a publicar anualmente, pudiera hacer las veces de dosier de prensa habitual ya que cumple una función análoga, tal y como se reseñaba en el marco teórico.

Finalmente, en quinto y último lugar, la tabla 4 permite observar claramente los resultados derivados de la aplicación del índice para la determinación de los públicos que se describía en el apartado metodológico.

Estos datos evidencian que el principal público destinatario de las acciones llevadas a cabo por la organización objeto de estudio es la opinión pública general, que representa el 39,07 $\%$ del total de públicos identificados a través del análisis. A este dato le sigue la prensa, con un $13,98 \%$ (incluyendo a la prensa general, la prensa especializada, la prensa nacional y la extranjera), el público interno $(10,57 \%)$, el sector profesional de la hostelería $(6,27 \%)$, los sumilleres $(2,86 \%)$ y los líderes de opinión $(2,69 \%)$.

\section{Conclusiones}

Atendiendo al marco teórico expuesto, la gestión de la comunicación organizacional puede explicarse como un proceso de planificación estratégica de las relaciones públicas cuya finalidad es establecer un clima de confianza a través del cual la organización persigue la confluencia de sus intereses corporativos con los intereses de los públicos de su entorno, y todo ello en pos de perseguir un beneficio mutuo.

Programar este proceso, implica identificar y seguir una serie de fases que, según los autores consultados, van desde la detección de una alteración en el equilibrio hasta entonces existente entre los intereses de una organización con los de sus públicos, el diagnóstico de dicho desequilibrio a través de la fase de investigación y su resolución a través del diseño estratégico de mensajes, estrategias y tácticas para solucionarlo.

Es aquí donde se advierte el importante papel que las técnicas específicas de gestión de las relaciones con los públicos juegan en dicho proceso ya que el empleo de estas técnicas 
permiten vehicular, de la forma más adecuada, aquellos mensajes corporativos estratégicamente diseñados a los públicos clave identificados en cada programa de gestión de la comunicación organizacional.

Cierto es que las estrategias y las tácticas son elementos fundamentales del proceso de planificación estratégica, que se fundamenta en la dirección por objetivos, pero ni una ni otra podrían llevarse a cabo sin las herramientas apropiadas, es decir, sin las diversas técnicas de relaciones públicas identificadas: técnicas de relaciones internas, técnicas de relaciones con la comunidad, técnicas transversales y, por supuesto las técnicas de relación con los medios de comunicación.

En concreto, las técnicas de relación con los medios de comunicación social, son aquellas herramientas encaminadas a generar un clima de entendimiento y beneficio mutuo entre la organización y la prensa con el objetivo de dar voz a la organización ante el propio medio de comunicación pero, sobre todo, ante la opinión pública. Su finalidad, por tanto, es situar en la agenda mediática y social el punto de vista de la organización a través de determinados mensajes corporativos estratégicamente predefinidos.

Desde esta perspectiva, la revisión de la literatura clásica en materia de relaciones públicas, así como los resultados del análisis, nos permiten establecer una conexión entre las técnicas referenciadas con mayor frecuencia por los autores consultados y las que realmente se llevan a cabo con mayor frecuencia a la hora de gestionar la comunicación organizacional. Es decir, la revisión conceptual y los datos derivados del análisis coinciden en identificar la nota de prensa, la rueda o conferencia de prensa y otros encuentros con la prensa (como las catas dirigidas o la gestión de entrevistas) como las técnicas de relación con los medios de comunicación más relevantes. Si bien es verdad que el análisis revela que el CRBJ no utiliza con frecuencia el dossier de prensa, que si es señalado por Xifra (2007: 148) como uno de los instrumentos relevantes para proporcionar información sobre la organización a los medios de comunicación aunque, tal y como señalábamos en el análisis, este dato podría deberse a que se utilice la memoria anual del CRBJ como material para que la prensa pueda ampliar información objetiva y detallada sobre la organización. En este contexto cabría reseñar que la memoria, aun cumpliendo en este sentido una función análoga a la del dosier, no puede definirse nunca como una técnica específica de relación con los medios de comunicación social sino como una técnica transversal (Xifra, 2007: 274-282).

Asimismo, este trabajo nos permite analizar el comportamiento comunicacional del CRBJ para determinar en qué grado se utilizan las técnicas de relación con los medios de comunicación frente a otras técnicas de relaciones públicas y, si es posible contemplar a los periodistas como públicos específicos a la hora de gestionar la comunicación de la organización objeto de estudio. 
En relación a la primera cuestión, tal y como nos demuestran los datos obtenidos en el análisis, podemos afirmar que las técnicas de relación con los medios de comunicación son un pilar importante para la gestión de la comunicación organizacional, pero no es el único y ni siquiera es el principal. Este dato refuerza empíricamente las aproximaciones de Grunig (2001) y Castillo (2010) que destacábamos al comienzo del trabajo y que coincidían en dar a las técnicas de relación con la presa un lugar justo entre todas las técnicas de comunicación y relaciones públicas que una corporación utiliza en sus planificaciones estratégicas de gestión de la comunicación. Efectivamente, nuestro estudio revela que las técnicas de relaciones públicas más utilizadas por el CRBJ son las técnicas de relación con la comunidad y las técnicas transversales, mientras que las técnicas de relación con los medios de comunicación social se ubican en el tercer lugar.

En relación a la segunda cuestión, el análisis revela que el público principal al que se dirige el CRBJ es la opinión pública general, siendo la prensa el segundo público en importancia, pero con una diferencia significativa de porcentaje. En este sentido, atendiendo a los objetivos inicialmente propuestos, es destacable que la prensa se erige como un destinatario relevante en el comportamiento comunicacional del CRBJ, pero no el único ni el principal.

La conclusión final de este trabajo, que consideramos de gran aplicabilidad en la praxis de la gestión de la comunicación en el ámbito profesional, es que las técnicas de relación con la prensa son una parte relevante a la hora de destinar recursos económicos y humanos en la gestión de la comunicación de las organizaciones. No obstante, pese a la visibilidad de estas técnicas, el peso de la gestión de la comunicación en las planificaciones estratégicas de las relaciones públicas no puede ni debe recaer en ellas. Un esfuerzo planificado y estratégico encaminado a buscar el entendimiento mutuo entre la organización y su entorno debe utilizar un amplio registro de técnicas que permitan vehicular de la forma más eficaz (rentabilizando canales y públicos) los mensajes corporativos a los stakeholders.

\section{REFERENCIAS BIBLIOGRÁFICAS}

ALMANSA, Ana (2005): "La comunicación organizacional en España: un camino a medio recorrer". Comunicación organizacional. Teorías y estudios. Málaga, Editorial Clave.

BARDIN, Laurence (1986): Análisis de contenido. Madrid, Akal Universitaria.

BERNAYS, Edward L. (1998): Cristalizando la opinión pública. Barcelona, Gestión 2000.

BOTAN, Carl y HAZLETON, Vincent (2006): Public Relations Theory II. Mahwah, New Jersey, Lawrence Earlbaum Associates, Inc.

CASTILLO, Antonio (2010): Introducción a las relaciones públicas. Málaga, IIRP.

CEA D ANCONA, María Ángeles (2001): Metodología cuantitativa. Estrategias y técnicas de investigación social. Madrid, Síntesis sociología.

CORBETTA, Perrigiorgio (2007): Metodología y técnicas de investigación social. Madrid, Mc Graw Hill. 
CUTLIP, Scott M. y CENTER, Allen H. (2001): Relaciones públicas eficaces. Barcelona, Gestión 2000.

FERNÁNDEZ LÓPEZ, Sergio (2007): Cómo gestionar la comunicación en organizaciones públicas y no lucrativas. Madrid, Narcea ediciones.

GONZÁlEZ RÍO, María J. (1997): Metodología de la investigación social. Técnicas de recolección de datos. Alicante, Aguaclara.

GRUNIG, James E. y HUNT, Todd (2000): Dirección de relaciones públicas, Barcelona, Gestión 2000.

KRIPPENDORFF, Klaus. (1990): Metodología del análisis de contenido. Teoría y práctica. Barcelona, Paidós Comunicación.

LEDINGHAM, John A. (2006): "Relationship management: a general theory of public relations". Public Relations Theory II. Lawrence Earlbaum Associates, Inc. Mahwah, New Jersey.

L'ETANG, Jacquie y PIECZKA, Magda (2006): Public Relations. Critical debates and contemporary practice. Mahwah (New Jersey), Lawrence Earlbaum Associates, Inc.

NOGUERO, Antonio (1988): Programación y técnicas de relaciones públicas. Barcelona, ESRP.

NOGUERO, Antonio (1995): La función social de las relaciones públicas: historia, teoría y marco legal. Barcelona, ESRP.

OTERO ALVARADO, María Teresa (2000): Teoría y estructura del ceremonial y el protocolo, Sevilla, Mergablum edición y comunicación.

OTERO ALVARADO, María Teresa (2009): Protocolo y organización de eventos. Barcelona, Ed. UOC.

PÉREZ SERRANO, Gloria (2004): Investigación cualitativa. Retos e interrogantes. Madrid, Editorial La Muralla.

PÉREZ SERRANO, Gloria (2007): Modelos de investigación cualitativa en educación social y animación sociocultural. Aplicaciones prácticas. Madrid, Narcea.

SELLTIZ, Claire, WRIGHTSMAN, Lawrence S., COOK, Stuart W. (1980): Métodos de investigación en las relaciones sociales. Madrid, Ediciones Rialp.

VAN RIEL, Cornelis B. Maria (2001): Comunicación corporativa. Madrid, Prentice Hall

WILCOX, Dennis L., AUTT, Philips H., AGEE, Warren K., y CAMERON, Glen T. (2000): Relaciones públicas. Estrategias y tácticas. Madrid, Pearson Addison Wesley.

WILCOX, Dennis L., CAMERON, Glen T. y XIFRA, Jordi (2009): Relaciones públicas. Estrategias y tácticas. Madrid, Pearson Addison Wesley.

XIFRA, Jordi (2003): Teoría y estructura de las relaciones públicas. Madrid, Mc Graw Hill.

XIFRA, Jordi (2005): Planificación estratégica de relaciones públicas. Barcelona, Paidós.

XIFRA, Jordi (2007): Técnicas de las relaciones públicas. Barcelona, Editorial UOC.

XIFRA, Jordi (2011): Manual de relaciones públicas e institucionales. Barcelona, Tecnos.

[Recibido: 21 de julio de 2015. Aceptado: 22 de octubre de 2015] 